\title{
Cognitive computing for intelligent application and service
}

\author{
Yin Zhang ${ }^{1} \cdot$ Haider Abbas $^{2} \cdot$ Yujie $\mathrm{Li}^{3}$ \\ Published online: 30 March 2020 \\ (C) Springer-Verlag London Ltd., part of Springer Nature 2020
}

Cognitive computing breaks the boundary between two separate fields, neuroscience, and computer science. It paves the way for machines to have reasoning abilities which is analogous to humans. The research field of cognitive computing is interdisciplinary and uses knowledge and methods from many areas such as psychology, biology, signal processing, physics, information theory, mathematics, and statistics. The development of cognitive computing will keep cross-fertilizing these research areas. However, in information applications and services there remain many open problems for cognitive computing. Technologies like cloud computing and big data are essential to upgrade the existing systems with near-human intelligence by using new capabilities such as machine learning, cognitive sensing, data mining, pattern recognition, and natural language processing.

This Special Issue features 11 selected papers with high quality that describe state-of-the-art technologies and new findings. The first article, "An LBP encoding scheme jointly using quaternionic representation and angular information," proposed a novel local descriptor, called the quaternionic local angular binary pattern (QLABP), for color image classification. QLABP was based on the quaternionic representation (QR) of color images such that it was able to handle all color components holistically as well as consider their relations. Using QR, the quaternionic angular information was further developed to account for more color characteristics.

The second article titled "Toward cognitive support for automated defect detection" proposed a neighborhood-

Yin Zhang

yin.zhang.cn@ieee.org

1 School of Information and Communication Engineering, University of Electronic Science and Technology of China, Chengdu, China

2 R\&D Department, Military College of Signals, NUST, Rawalpindi, Pakistan

3 Yangzhou University, Yangzhou, China maintaining approach, which was based on the minimum ratio for fast and reliable inspection of industrial products. The minimum ratio between local neighborhood sliding windows was used as a similarity measure for localizing defection.

In the next article with the title "Distributed representation learning via node $2 \mathrm{vec}$ for implicit feedback recommendation," the authors proposed a deep learningenhanced framework for implicit feedback recommendation. In this framework, it simultaneously learned the new distributed representation of users and items via node2vec to improve the negative sampling strategy. Finally, a deep neural network recommendation model was developed to integrate user features, product features, and interaction features.

The existing solutions for wireless medical devices are either heavy for memory-constrained devices or require additional devices to be worn. The fourth article titled "Multi-layer security scheme for implantable medical devices" proposed a novel electrocardiogram authentication scheme which uses Legendre approximation coupled with a multi-layer perceptron model for providing three levels of security for data, network and application levels. The proposed model could reach up to $99.99 \%$ testing accuracy in identifying the authorized personnel even with 5 coefficients.

The fifth article titled "Face image super-resolution with pose via nuclear norm regularized structural orthogonal Procrustes regression" proposed a nuclear norm regularized structural orthogonal Procrustes regression (N2SOPR) approach in this work to acquire pose-robust feature representations for face hallucination with a pose. The orthogonal Procrustes regression was used to seek an appropriate transformation between two data matrixes. Additionally, the nuclear norm regularization was imposed on the representation residual to preserve image structural property.

In the sixth article, "Lung cancer prediction using higher-order recurrent neural network based on glowworm swarm optimization," a higher-order neural network 
system called recurrent neural network with LevenbergMarquardt model with the help of glowworm swarm optimization algorithm was proposed for managing multimodal disease information. The execution of the proposed strategies was tried with information and the benchmark dataset, and the outcomes demonstrate that the higher-order recurrent neural systems with glowworm swarm optimization gave better accuracy of $98 \%$ in comparison with a customary optimized neural network.

In the next article with the title "How textual quality of online reviews affect classification performance: a case of deep learning sentiment analysis," the authors explored the impact of two influential textual features, namely the word count and review readability, on the performance of sentiment classification. Three representative deep learning techniques, namely SRN, LSTM, and CNN, were applied to sentiment analysis tasks on a benchmark movie reviews dataset, while multiple regression models were further employed for statistical analysis.

Data analytics, with its promise to efficiently discover valuable patterns by analyzing a large amount of unstructured, heterogeneous, non-standard and incomplete healthcare data. The eighth article, "Big data analytics for preventive medicine" provided a comprehensive and structured overview of extensive research on the advancement of data analytics methods for disease prevention, and summarized state-of-the-art data analytics algorithms used for classification of disease, clustering (unusually high incidence of a particular disease), anomalies detection (detection of disease) and association as well as their respective advantages, drawbacks, and guidelines for selection of specific model followed by a discussion on recent development and successful application of disease prevention methods.

The ninth article titled "A pricing method of online group-buying for continuous price function" adopted an algorithm called Vickrey-Clarke-Groves for group-buying; buyers' payments were the sum of commodities' price and the extra amount by purchase quantity. The mechanism motivated buyers to report truthful preference through the compensatory payment.

In the eleventh article, "Intelligent equipment design assisted by Cognitive Internet of Things and industrial big data," the authors proposed an innovative design method to help design intelligent equipment. Firstly, referring to the architecture of the Cognitive Internet of Things (CIoT) and industrial big data, it proposed the architecture of the method and defined the different layers to process the data. Then, for the acquired external data, it put forward an algorithm which was combined with the technology of CIoT and industrial big data, to help designers analyze and make decisions.

The last article titled "High-quality-guided artificial bee colony algorithm for designing loudspeaker" proposed a high-quality-guided artificial bee colony algorithm to increase its convergence speed and search accuracy by gradually changing the number of updating dimensions and searching closer to better locations. The algorithm was first tested on some representative basic benchmark functions and then was applied to a loudspeaker design problem.

In conclusion, the leading editor and the guest editors would like to thank all the authors who submitted their research articles to our Special Issue. We highly appreciate the contributions of the reviewers for their constructive comments and suggestions. We also would like to acknowledge the guidance from the Editor-in-Chief and staff members.

Publisher's Note Springer Nature remains neutral with regard to jurisdictional claims in published maps and institutional affiliations. 